\title{
Foreword to special issue on homocysteine disorders
}

\author{
Brian Fowler • Henk J. Blom • Viktor Kožich
}

Published online: 11 January 2011

(C) The Author(s) 2011. This article is published with open access at Springerlink.com

This special issue brings together a series of articles resulting from two recent symposia which focussed on homocysteine metabolism and related disorders.

The 7th International conference on homocysteine metabolism took place in Prague from the 21st to the 25th of June, 2009. This conference was the latest in the series following those held in Dromoland Castle Co. Clare, Ireland in 1995, in Nijmegen, the Netherlands in 1998, in Sorrento, Italy in 2001, in Basel, Switzerland in 2003, in Milan, Italy in 2005 and in Saarbrücken, Germany in 2007. In 2011 this meeting is planned to take place in Lisbon, Portugal. This conference tries to link basic science with clinical applications in the field of homocysteine, a field which has seen a dramatic increase in interest over the last 20 years. Current issues were addressed in Plenary sessions on: metabolites and their trafficking; genes; patho-physiology of disturbed C-1 and sulphur amino acid pool; 3 free communication sessions; 2 sessions on lessons from intervention trials and fortification.

Two articles bring to attention two relatively poorly addressed pathways which could well be of increasing future importance. The extensive review by Ueland (doi:10.1007/ s10545-010-9088-4) on choline and betaine emphasises the

\author{
B. Fowler $(\bowtie)$ \\ Basel, Switzerland \\ e-mail: h.blom@vumc.nl \\ H. J. Blom ( $\square)$ \\ Metabolic Unit, Department of Clinical Chemistry, \\ Institute for Cardiovascular Research (ICaR-VU), \\ VU University Medical Centre, \\ Amsterdam, The Netherlands \\ e-mai: h.blom@vumc.nl \\ V. Kožich \\ Prague, Czech Republic \\ e-mail: Viktor.kozich@1f1.cuni.cz
}

importance of these metabolites in essential processes and recent studies on their possible links to chronic disease. The review by Stipanuk on the metabolic processes between cysteine and taurine and inorganic sulphur highlights that homocysteine can be a "good guy" in acting as precursor of this important pathway (doi:10.1007/s10545-010-9006-9). The key enzyme in homocysteine catabolism, cystathionine $\beta$-synthase, is central to three articles. The paper by Magner et al. (doi:10.1007/s10545-010-9146-y) points out a changing clinical spectrum of cystathionine synthase deficiency as patients with a mainly vascular disease pathology are discovered partly as a result of the recent focus on mild hyperhomocysteinaemia as a risk factor. Current focus on chaperone effects on this enzyme are highlighted in the paper of Kopecka and colleagues (doi:10.1007/s10545-0109087-5). A new approach to the assay of cystathionine synthase using tandem MS/MS somewhat surprisingly shows that this enzyme is detectable in plasma with diagnostic implications (doi:10.1007/s10545-010-9178-3).

The transport of homocysteine has received relatively little attention and therefore the paper by Glazier et al. (doi:10.1007/ s10545-010-9141-3) is especially welcome with potential implications for the role of homocysteine in pregnancy.

There is always great interest in epidemiological aspects of homocysteine as a risk factor at such meetings and the report of the large multi-centre study on the effect of vitamin intervention on the secondary occurrence of vascular disease was greatly anticipated. The largely negative results are disappointing but some authors question the extrapolation of these findings to exclude possible benefits on primary events.

The controversial issue of cobalamin fortification of food is addressed in the article by Carmel (doi:10.1007/s10545010-9150-2). This author argues against fortification, certainly at the present time but it must be borne in mind 
that there is a counter argument within the overall context of B vitamin fortification of foodstuffs and hopefully this article will contribute to debate on this topic.

The annual international meeting on inborn errors of metabolism organised in Fulda, Germany by the SHS company brings together clinicians and basic scientists to explore advances and their implications for treatment and outcome in patients with rare inborn errors of metabolism. We were very pleased that for this year it was decided to revisit the topic "Homocysteine, Folate and Cobalamin disorders" just 13 years after it first appeared.

The meeting was opened with an extensive review of related pathways with reference to vascular disease and neural tube defects reported in the article by Henk Blom (doi:10.1007/s10545-010-9177-4).

We were fortunate that Robert Clarke repeated his presentation from Prague here (doi:10.1007/s10545-0109235-y). In addressing the so called homocysteine controversy Yvo Mulders (doi:10.1007/s10545-010-9151-1) raises several possible limitations on the previous intervention studies that seem plausible and which might moderate the mainly negative conclusions and he convincingly argues that homocysteine is far from dead. The paper by Susan Duthie on "Folate and cancer: how DNA damage, DNA repair and DNA methylation impact on colon carcinogenesis" (doi:10.1007/s10545-010-9128-0) is a valuable and very informative review of one example of the possible involvement homocysteine and folate in a common disease.

The current interest in vitamin $\mathrm{B}_{12}$ and its disorders is reflected by five related articles.

The presentation on cobalamin status in the general childhood population reported an approach to nutritional studies that is unfamiliar to most paediatricians in the inborn errors field (doi:10.1007/s10545-010-9119).

Inborn errors related to cobalamin are well represented with four papers.
One of the several transport defects, transcobalamin deficiency is covered in the article by Nissen from the group of Ebba Nexo which reports the complexity of mutation analysis in this disorder (printed as abstract only, doi:10.1007/s10545-010-9145-z).

The elegant studies leading to the discovery of the gene for the cblF defect of intracellular metabolism which were rather surprisingly based on homozygosity mapping in several unrelated families are reported by Rutsch (doi:10.1007/s10545-010-9083-9).

A comprehensive review of clinical presentation and outcome of the $\mathrm{Cbl} \mathrm{C}$ defect by Martinelli et al. emphasises pathogenetic aspects and sets the scene for the future studies necessary to try to improve outcome in this difficult to treat disorder (doi:10.1007/s10545-010-9161-z).

The question of impact of treatment on the remethylation defects is extended to other disorders by Schiff et al. calling on the vast experience from one main centre involved in care of such patients (doi:10.1007/s10545-010-9120-8).

Finally the paper by Rozen brings out the elegant, detailed studies that are possible in appropriate animal models, in this case for deficiency of the key folate enzyme, methylenetetrahydrofolate reductase (doi:10.1007/s10545-010-9127-1).

All in all the variety of papers in the special issue emphasise the importance of homocysteine as a key metabolite that is linked to several basic biological processes and therefore not surprisingly involved in these several disease processes.

Brian Fowler

With thanks to my co editors, Henk Blom and Viktor Kožich

Open Access This article is distributed under the terms of the Creative Commons Attribution Noncommercial License which permits any noncommercial use, distribution, and reproduction in any medium, provided the original author(s) and source are credited. 